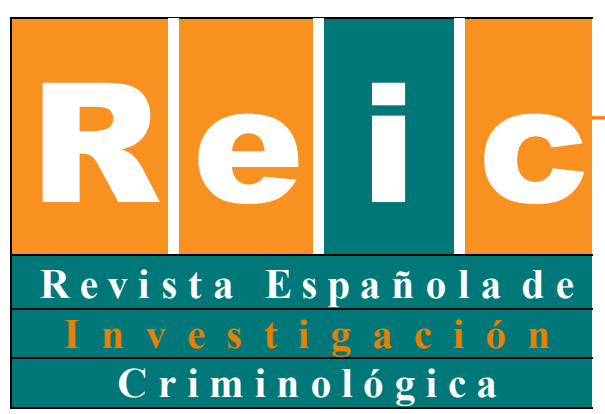

Aizpurúa

\title{
Presente y futuro del estudio de la opinión pública hacia el castigo de los menores infractores. Evidencias, carencias y posibilidades
}

\section{Present and future of the study of public opinion toward the punishment of juvenile offenders. Evidence, shortcomings and opportunities}

Recibido el 7 enero 2014/Publicado el 7 abril 2014

\author{
Eva Aizpurúa ${ }^{1}$ \\ Universidad de Castilla-La Mancha
}

\begin{abstract}
RESUMEN
Las últimas décadas han presenciado la deriva punitiva que ha experimentado la justicia de menores. Paradójicamente, este desplazamiento no ha obedecido a un aumento de la delincuencia juvenil, sino que ha sido justificado, en gran medida, recurriendo a las demandas sociales. La repercusión que ha alcanzado la opinión pública en la reciente política criminal la ha convertido en una de las principales líneas de investigación de la criminología actual. Por ello, el presente trabajo tiene como objeto sistematizar y discutir las evidencias científicas nacionales e internacionales, identificando las limitaciones y retos que enfrenta este ámbito de estudio. En un primer momento, se describen los elementos característicos de las actitudes y se analizan sus principales factores explicativos. Posteriormente se revisan los abordajes metodológicos utilizados, examinando sus posibilidades e inconvenientes más importantes. Finalmente, se valoran las implicaciones teóricas y metodológicas que se desprenden del trabajo, aportando elementos significativos para su desarrollo futuro.
\end{abstract}

\footnotetext{
${ }^{1}$ La correspondencia debe enviarse a: Eva Aizpurúa González. Centro de Investigación en criminología. Universidad Castilla-La Mancha. Edificio Benjamín Palencia. Campus Universitario, s/n. 02071 Albacete (España).Eva.Aizpurua@uclm.es
} 
Palabras clave. Delincuencia juvenil, justicia de menores, opinión pública, punitivismo, técnicas de investigación.

\begin{abstract}
In recent decades there has been a punitive turn in the area of juvenile justice. Paradoxically, this shift has not come about as a result of an increase in juvenile crime, but rather has been justified, to a large extent, by citing public opinion. The impact of social demands on criminal policy has led to it becoming one of the main lines of research in criminology today. This paper seeks to systematize and discuss national and international scientific evidence in order to identify the constraints and challenges facing this field of study. Firstly, the characteristic features of attitudes towards juvenile criminality are described and the explanatory factors are analyzed. Subsequently, the article reviews the methodological approaches which have been used to measure public opinion, examining their most significant strengths and failings. Finally, it evaluates the theoretical and methodological implications of this paper, providing insights for the future development of this area of research.
\end{abstract}

Keywords. Juvenile delinquency, juvenile justice, public opinion, punitivism, research techniques.

\title{
1. Introducción
}

La investigación referida a los aspectos delincuenciales focalizó su atención, en un primer momento, en los infractores y, posteriormente, en los agentes de control social. Sólo recientemente ha dirigido su análisis a la ciudadanía, a las respuestas que ésta reclama ante las transgresiones legales y a su repercusión en las decisiones políticocriminales. Ciertamente, no fue hasta la década de 1990 cuando se generalizaron los estudios tendentes a conocer las percepciones y actitudes de la población hacia las infracciones cometidas por menores. No obstante, las transformaciones en las respuestas adoptadas frente al delito juvenil -que se han materializado en una intensa actividad de reforma (Allen, 2002; Bishop, 2006; McAuley y McDonald, 2007; Rechea, Fernández y Benítez, 2004)-, unidas al cambio que se ha producido en el lugar que ocupa la delincuencia en nuestra cotidianeidad e imaginario colectivo (Garland, 2001), han convertido esta materia en uno de los principales ámbitos de estudio de la criminología actual (Roberts, 2005; Varona, 2008). 
Concretamente, puede afirmarse que el auge de este campo teórico ha obedecido a dos motivos fundamentales. El primero de ellos es la resonancia que ha alcanzado la opinión pública en las decisiones político-criminales (Jan, Ball y Walsh, 2008; Roberts y Hastings, 2007). Un hecho que ha encontrado reflejo, en nuestro contexto más inmediato, en las sucesivas reformas que ha experimentado la Ley Orgánica 5/2000, reguladora de la responsabilidad penal de los menores (en adelante, LO 5/2000), donde la mayoría de las iniciativas no han respondido a una ruptura en la evolución de la criminalidad juvenil, sino que se han practicado con la pretensión de satisfacer las demandas sociales y responder al modo en que la ciudadanía reacciona ante los delitos cometidos por menores. Así lo demuestra la Exposición de Motivos de la Ley Orgánica 8/2006, modificadora de la LO 5/2000, cuando puntualiza que "no han aumentado significativamente los delitos de carácter violento, aunque los realmente acontecidos han tenido un fuerte impacto social", e inmediatamente después, articula una reforma que se tradujo en una mayor severidad de las consecuencias jurídicas previstas para los menores infractores, privilegiando la preocupación social por la delincuencia violenta en detrimento de su realidad estadística (Guanarteme, 2009).

En segundo lugar, cabe recalcar que el estudio de la opinión pública constituye una tarea natural en el marco de una sociedad democrática. En este sentido, la confluencia de las demandas sociales con los principios que rigen el sistema penal es el fundamento indispensable para garantizar su legitimidad y correcto funcionamiento (Hohl, Stanko y Newburn, 2013; Roberts, 2004). Ello es así porque las teorías desarrolladas durante las últimas décadas, particularmente las formuladas por Tyler (2003), han revelado cómo el cumplimiento de las normas viene determinado por su aceptación social. De modo que cuando la población percibe a los tribunales como organismos legítimos, tiende a obedecer, en mayor medida, los preceptos que emanan de ellos y a cooperar con la justicia, en tareas como denunciar los delitos y colaborar en calidad de testigos.

A diferencia de otros países occidentales en los que esta línea de investigación se encuentra firmemente arraigada, en España el análisis de las actitudes punitivas constituye una esfera incipiente y poco explorada. Si bien se han incrementado notablemente los estudios de opinión pública hacia la delincuencia adulta y sus instituciones de control (García, 2013; Marteache, 2012; Marteache, Martínez y Pérez, 
2010; Serrano, 2011; Varona, 2008, 2013), son escasos los trabajos que han evaluado las actitudes hacia el delito juvenil. Desde que Fernández y Tarancón realizasen la primera aproximación empírica en 2010, se han publicado tres investigaciones, que comprenden la efectuada por García, Martín, Torbay y Rodríguez (2010) referida al conocimiento y la valoración ciudadana de la LO 5/2000, el cuasi-experimento conducido por Aizpurúa y Fernández (2011) que examina el efecto de la información en las preferencias hacia el castigo juvenil y el reciente trabajo de Fernández (2013), en el que se analizan las exigencias punitivistas de la sociedad española en el ámbito de la justicia de menores.

El restringido desarrollo de este cuerpo teórico intensifica la necesidad de incrementar las investigaciones en la materia. La evidencia acumulada ha mostrado cómo la edad desempeña un rol fundamental en la explicación del punitivismo, revelando cómo la población reclama respuestas más rigoristas cuando los infractores son adultos (Piquero, Cullen, Unnever, Piquero y Gordon, 2010; Roberts, 2004; Tufts y Roberts, 2002). Dado el potencial de la edad para modular las preferencias ciudadanas, resulta esencial profundizar en el conocimiento de las actitudes hacia el castigo juvenil. Con la finalidad de contribuir a ello, este artículo responde al doble propósito de sistematizar los principales hallazgos científicos y aportar elementos significativos para su desarrollo teórico y metodológico. Su interés radica en dos circunstancias fundamentales que comprenden la abundancia y la dispersión de la producción científica. Si bien existen algunas revisiones teóricas publicadas en lengua inglesa (entre las cuales cabe destacar las elaborados por Halsey y White, 2008 y por Roberts, 2004), no ocurre lo mismo en nuestro contexto, siendo ésta la razón de ser y principal aportación de este trabajo. A través de él se procurará establecer una base preliminar que ofrezca una perspectiva global del estado de la cuestión e identifique aquellas áreas de incertidumbre que precisan de un mayor conocimiento. 


\section{Rasgos distintivos de la opinión pública hacia el castigo de los menores infractores y el sistema de justicia juvenil}

Pese a las variaciones contextuales y metodológicas ${ }^{2}$, las investigaciones desarrolladas durante las últimas décadas han identificado ciertos rasgos característicos de las actitudes hacia la delincuencia juvenil y las sanciones aplicables a los menores infractores.

En primer lugar, coincidiendo con los hallazgos obtenidos en relación con la delincuencia adulta (Hough y Roberts, 1998; Varona, 2008), se ha encontrado un conocimiento distorsionado de las infracciones juveniles. Así, los estudios han revelado que la población alberga una percepción magnificada de la conducta delictiva de los jóvenes, tanto en términos cuantitativos -atendiendo a su frecuencia y alcance-, como en términos cualitativos -considerando su gravedad- (Haines y Case, 2007; Halsey y White, 2008). De este modo, se ha comprobado la tendencia a sobrestimar la proporción de delitos cometidos por menores (Haines, 2007; Hough y Roberts, 2004), considerando que éstos se encuentran en constante aumento (Hamilton y Harvey, 2005; Jones y Weatherburn, 2011), así como la predisposición a equiparar la criminalidad juvenil con los constructos de violencia y reincidencia (Gelb, 2006, 2008; Halsey y White, 2008). Tales resultados han sido una constante en las aproximaciones internacionales, permaneciendo invariables a pesar de la evolución de las tasas delictivas (Hamilton y Harvey, 2005; Hough y Roberts, 2004). Coexistiendo con ello, se ha encontrado una profunda insatisfacción con la justicia juvenil, que se ha traducido en la tendencia a calificar de indulgentes las medidas impuestas por los tribunales (Barber y Doob, 2004; Mears, Hay, Gertz y Mancini, 2007; Scott, Repucci, Antonishak y DeGennaro, 2006; Sprott, 1996; Varma, 2006). Al igual que ocurriera con las infracciones juveniles, la noción de benevolencia en el tratamiento de los menores se ha mantenido estable pese a la multiplicidad de reformas que han ido endureciendo la respuesta penal (Covell y Howe, 1996). No obstante, cabe advertir, como así lo han hecho diversos autores (entre ellos, Gelb, 2008; Hough y Roberts, 2004; Tufts y Roberts, 2002), que la ciudadanía recurre al imaginario de infractores violentos y reincidentes cuando critica la

\footnotetext{
${ }^{2}$ Que comprenden, entre otros aspectos, la desigualdad de los tamaños muestrales y análisis estadísticos practicados, la diversidad de técnicas de recogida de datos utilizadas, así como la desigual operacionalización de las variables empleadas en unas y otras aproximaciones.
} 
condescendencia del sistema de justicia juvenil. En esta misma línea, uno de los hallazgos más sólidos que se desprenden de la literatura comparada es la desigual confianza depositada en los distintos organismos que integran el entramado penal, siendo los juzgados de menores las instituciones peor valoradas del conjunto (Allan, Edwards, Patterson y Smith, 2006; Tufts, 2002).

Estos resultados han sido justificados aludiendo a tres factores, que comprenden el limitado conocimiento público de las tendenciales delictivas y el funcionamiento de la justicia juvenil (Chapman, Mirrlees-Black y Brawn, 2002; Haines, 2007; Hough y Roberts, 2004), la retórica política y la consiguiente instrumentalización del derecho penal a través de lo que se ha dado en llamar "populismo punitivo" (Bottoms, 1995), así como el potencial que poseen los medios de comunicación en la construcción del fenómeno, ofreciendo, a menudo, una representación deformada y fragmentaria del mismo (Estrada, 2001; Faucher, 2009).

Sin embargo, coincidiendo con estas evidencias, que pudieran ser indicativas de una población punitiva, existe un consistente grupo de hallazgos que refuerza el carácter ambivalente y polifacético de la opinión pública (Baron y Hartnagel, 1996; Haines y Case, 2007; Sprott, 1996). Las distintas aproximaciones han mostrado un pronunciado apoyo hacia las penas alternativas al internamiento (Gelb, 2006, 2008; Piquero y otros, 2010) y hacia la rehabilitación como objetivo nuclear de la justicia juvenil (Applegate, Davis y Cullen, 2009; Mears, 2001; Moon, Sundt, Cullen y Wright, 2000). Igualmente, se ha verificado que la población avala el tratamiento diferenciado de los menores (Piquero y otros, 2010; Scott y otros, 2006; Varma, 2006), respaldando el mantenimiento de un sistema penal propio, frente a un modelo basado, exclusivamente, en la proporcionalidad de la pena (Haines y Case, 2007; Mears y otros, 2007; Piquero y otros, 2010). Todo ello amparado por la premisa de que los jóvenes presentan mayor maleabilidad y receptividad a la rehabilitación que sus homólogos adultos (Moon y otros, 2000; Piquero y otros, 2010). No obstante, es destacable que estas posiciones se encuentran supeditadas a la naturaleza del delito (Tufts, 2002), como indica un cuerpo creciente de estudios que ha revelado que las conductas violentas y aquellas de especial gravedad establecen una clara ruptura entre las actitudes punitivas y no punitivas de la ciudadanía (Hamilton y Harvey, 2005, Jan y otros, 2008; Mays y Rudell, 2012; Steinberg y Piquero, 2010). 
En consonancia con Mascini y Houtman (2006) no existe una correspondencia entre el apoyo hacia la rehabilitación y la defensa del castigo, de modo tal que la población respalda objetivos y estrategias múltiples de forma simultánea y no excluyente, superando la dicotomía educación-castigo instaurada en la esfera académica (Aizpurúa y Fernández, 2011; Fernández y Tarancón, 2010).

Tal y como revela este conjunto de resultados, la inconsistencia preside, en gran medida, las relaciones existentes entre la delincuencia, la opinión ciudadana y la política criminal (Green, 2006). Por ello, cobra especial relevancia el término "comedia de errores" acuñado por Allen (2002) en referencia a la situación imperante, donde las políticas impulsadas no se fundamentan en una comprensión rigurosa de la opinión pública, al tiempo que la opinión pública no se asienta en un conocimiento preciso de la teoría y la práctica subyacentes.

\section{Los factores explicativos de la opinión pública hacia la el castigo de los menores infractores y el sistema de justicia juvenil}

En paralelo a la proliferación de estudios orientados a identificar los rasgos distintivos de la opinión pública en esta materia, comenzó a gestarse una línea de investigación que, trascendiendo el plano descriptivo, exploraba sus factores explicativos. Todo ello basado en la convicción de que la comprensión integral de las actitudes exigía el conocimiento de sus elementos constitutivos (Roberts y Indermaur, 2007). Así, las investigaciones desplegadas en la esfera internacional condujeron al establecimiento de cinco ejes principales vinculados con las actitudes punitivas, distinguiendo entre aquellas variables sociodemográficas, atribucionales, cognitivas, instrumentales y finalmente, expresivas, que poseían el potencial de modular los posicionamientos hacia el delito juvenil. 
Figura 1. Factores explicativos de las actitudes hacia el castigo

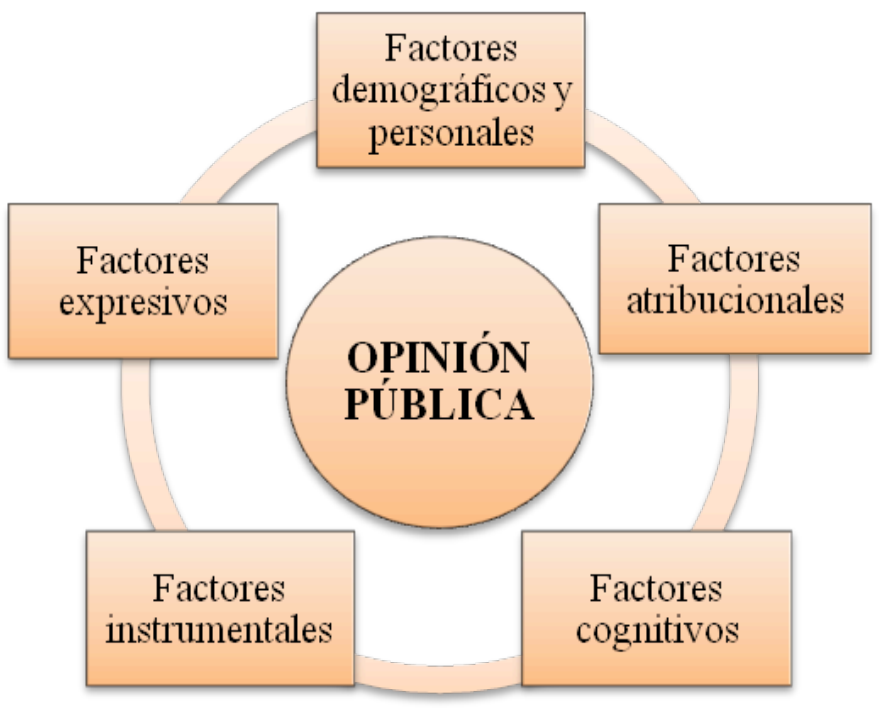

Fuente: Elaboración propia a partir de Gelb (2008) y Roberts y Indermaur (2007).

\section{a. Variables demográficas y personales}

Las primeras aproximaciones examinaron los predictores demográficos de la opinión pública, analizando factores como la edad, el sexo, el origen étnico o el nivel formativo de los encuestados (Roberts y Indermaur, 2007).

El hallazgo más sólido que se desprende de ellas es la existencia de una correlación inversa entre el nivel de estudios y las actitudes punitivas (Baron y Hartnagel, 1996; Gelb, 2006; King y Maruna, 2010; Mahew y Van Kesteren, 2002; Mears, 2001; Piquero y otros, 2010; Tufts, 2002; Wu, 2000), siendo la educación el predictor demográfico más significativo de la opinión pública en esta materia (Gelb, 2011; Maruna y King, 2004; Payne, Gainey, Triplett y Danner, 2004; Roberts y Indermaur, 2007; Spiranovic y otros, 2012).

Resultados heterogéneos se han cosechado con relación a la edad. Mientras que algunos estudios han encontrado que el sentimiento punitivo se intensifica entre los sujetos más mayores (Baron y Hartnagel, 1996; Haines y Case, 2007; Jan y otros, 2008; Payne y otros, 2004; Wood y Vicki, 2004; Wu, 2000), otros han certificado o bien lo opuesto (Applegate y Davis, 2006; Covell y Howe, 1996; Haines, 2007; Piquero y otros, 2010), o bien la existencia de una relación curvilínea entre ambos extremos (Mears, 
2001; Schwartz, Guo y Kerbs, 1993). No obstante, a pesar de que los hallazgos son contradictorios y no permiten el establecimiento de conclusiones categóricas, el peso de la evidencia tiende a apoyar que el incremento de la edad se vincula con posicionamientos de carácter retributivo.

En lo que respecta al sexo, salvo limitadas excepciones (Guetti y Redlich, 2001; Payne y otros, 2004; Tufts, 2002) la investigación ha revelado la mayor tendencia de los hombres a mantener posicionamientos punitivos (Baron y Hartnagel, 1996; Haines, 2007; Mahew y Van Kesteren, 2002; Mears, 2001; Mears y otros, 2007; Perelman y Clements, 2009; Roberts y Indermaur, 2007; Schwartz y otros, 1993; Sprott, 1999; Wu, 2000), así como su menor tendencia a apoyar tesis rehabilitadoras (Piquero y otros, 2010). Estos contrastes han sido explicados aludiendo a la socialización diferencial de género $\mathrm{y}$, en concreto, a las diferencias que presentan mujeres y hombres en el constructo de empatía (Jan y otros, 2008; Sharp, McGhee y Hope, 2007; Wood y Vicki, 2004).

Además de los rasgos demográficos, los expertos han explorado la influencia de los factores personales sobre las actitudes hacia el castigo, observando el impacto de variables como la orientación política y la adscripción religiosa de los participantes. En este sentido, la literatura ha revelado que la ideología conservadora correlaciona, de forma positiva y significativa, con la defensa de postulados retributivos (Applegate y Davis, 2006; Baron y Hartnagel, 1996; Hartnagel y Templeton, 2012; King y Maruna, 2010; Mears y otros, 2007; Roberts y Indermaur, 2007; Scott y otros, 2006; Steinberg y Piquero, 2010; Wu, 2000). Estos hallazgos han sido explicados aludiendo a la vinculación de la tendencia política con los estilos atribucionales, habiéndose encontrado que quienes albergan una ideología conservadora presentan, en mayor medida, estilos de atribución clásicos (Carmichael y Burgos, 2012; Cochran, Boots y Heide, 2003; Grasmick y McGill, 1994), cuya vinculación con la punitividad ha sido ampliamente verificada (Gelb, 2008; Maruna y King, 2004; Perelman y Clements, 2009).

En lo que a creencias religiosas se refiere, los resultados han sido dispares, cabiendo distinguir entre quienes se adhieren una práctica fundamentalista y quienes defienden una lectura flexible de la doctrina (Evans y Adams, 2003; Roberts y Indermaur, 2007). Sin embargo, la mayoría de investigaciones han hallado que las 
creencias religiosas actúan como catalizadores de las actitudes punitivas (Aizpurúa y Fernández, 2011; Wu, 2000). Al igual que ocurriera con la orientación política, la relación entre ambos extremos estaría mediada por el estilo atribucional (Grasmick y McGill, 1994).

En todo caso, los datos analizados muestran la naturaleza compleja y a menudo contradictoria de las vinculaciones existentes entre las variables demográficas y personales y la opinión pública hacia el castigo juvenil, cuya capacidad explicativa ha sido limitada, teniendo en cuenta los resultados obtenidos. En consonancia con ellos, las primeras tendrían el potencial de explicar entre un $7 \%$ y un $15 \%$ de la varianza en las actitudes (Gelb, 2011; King y Maruna, 2010; Maruna y King, 2004; Payne y otros, 2004; Roberts y Indermaur, 2007; Spiranovic y otros, 2012), mientras que las segundas esclarecerían en torno al 10\% de esta varianza (King y Maruna, 2010; Roberts y Indermaur, 2007), comportando, en uno y otro caso, tamaños de efecto pequeños en las investigaciones de esta índole (Cohen, 1998).

\section{b. Variables atribucionales}

Tal y como ha revelado la literatura, las percepciones se encuentran mediadas, también, por los procesos de atribución individuales. Siguiendo a Heider (1958, citado en Vaughman, 2010), las creencias atribucionales pueden estructurarse en dos grandes categorías. Las clásicas o disposicionales, según las cuales la delincuencia es interpretada como el producto de una opción individual, y las situacionales, que conciben el delito como el resultado de una variedad de circunstancias estructurales. En este sentido, la evidencia ha demostrado la conexión de las actitudes punitivas con los ideales clásicos (Evans y Adams, 2003; Gelb, 2008; Hartnagel y Templeton, 2012; Maruna y King, 2004; Perelman y Clements, 2009). Y ello es así porque quienes apoyan los principios disposicionales entendiendo el delito como una elección, tenderían a identificar a los infractores como responsables de sus acciones $\mathrm{y}$, en consecuencia, merecedores de respuestas punitivas. En contraste, aquellas personas que poseen creencias situacionales, considerando que los factores sociales son el germen de las conductas delictivas, mostrarían una menor inclinación por las medidas retributivas (Baron y Hartnagel, 1996; Grasmick y McGill, 1994; Piquero y otros, 2010). 
No obstante, como ocurriera con las variables sociodemográficas, los resultados muestran que los estilos atribucionales explican en torno al 10\% de la varianza en las actitudes (Cochran y otros, 2003; Maruna y King, 2004), suponiendo predictores débiles de las mismas (Cohen, 1998).

\section{c. Variables cognitivas}

Si bien los factores anteriores no proporcionaron una explicación integral de los elementos que vertebran las actitudes, los factores cognitivos han contribuido a ofrecer una mejor comprensión de ellos.

Así lo evidencia un cuerpo de estudios que ha explorado el impacto de la información sobre las opiniones y los juicios colectivos. Los resultados de estos trabajos han revelado cómo la opinión pública se encuentra condicionada por la calidad y la cantidad de información disponible. De este modo, se ha comprobado que la provisión de datos referidos al funcionamiento de la justicia penal y la prevalencia de la criminalidad no sólo redunda en un conocimiento más riguroso de ambos aspectos, sino que, al tiempo, determina las actitudes punitivas y la confianza depositada en el sistema, suscitando percepciones más positivas hacia el mismo (Aizpurúa y Fernández, 2011; Champan y otros, 2002; Hough y Park, 2002; Indermaur, Roberts, Spiranovic, MacKenzie y Gelb, 2012; Marteache y otros, 2010; Singer y Cooper, 2008; Quinton, 2011). Sin embargo, cabe apuntar, como así lo han hecho diversos autores (entre ellos, Chapman y otros, 2002; Quinton, 2011), que si bien existe una vinculación entre conocimiento y opinión ciudadana, esta es una asociación indirecta mediada por diversas variables, siendo la información una condición necesaria, pero no suficiente para generar cambios actitudinales perdurables (Green, 2006, 2012; Indermaur y otros, 2012).

Al tiempo que progresaban estas aproximaciones, fue emergiendo un grupo de investigaciones que examinó el efecto de las circunstancias concernientes al delito sobre las actitudes. Un enfoque que descansaba en el reconocimiento de que los planteamientos previos habían privilegiado el análisis de las variables relativas a los participantes en detrimento de las condiciones específicas del hecho. Fue así como se halló que las posiciones punitivas se encontraban subordinadas a la naturaleza violenta 
del delito (Applegate y Davis, 2006; Jan y otros, 2008; Piquero y Steinberg, 2010). En lo referido al infractor, se encontró que la defensa del castigo se asociaba con la mayor edad de los implicados (Applegate y Davis, 2006; Scott y otros, 2006; Spiranovic y otros, 2012; Steinberg y Piquero, 2010; Tufts, 2002; Tufts y Roberts, 2002), con su carácter reincidente (Applegate y Davis, 2006; Spiranovic y otros, 2012; Tufts, 2002; Tufts y Roberts, 2002) y su mayor grado de implicación en el delito (Garberg y Libkuman, 2009). Igualmente, recientes estudios han revelado cómo las actitudes hacia el castigo se encuentran vinculadas con las creencias hacia distintos aspectos del desarrollo adolescente. Concretamente, se ha observado que la presunción de la mayor impulsividad, vulnerabilidad a la presión de grupo y receptividad al tratamiento de los jóvenes previene los posicionamientos punitivos (Allen, Trzcinski y Kubiak, 2012; Trzcinski y Allen, 2012).

Este conjunto de factores ha mostrado que las circunstancias vinculadas al hecho delictivo y al infractor constituyen predictores significativos de la opinión pública, cuya capacidad explicativa ha oscilado entre el 7,5\% y el $30 \%$ de la varianza en las preferencias colectivas hacia el castigo juvenil (Spiranovic y otros, 2012; Steinberg y Piquero, 2010; Trzcinski y Allen, 2012).

\section{d. Variables instrumentales}

De conformidad con las tesis instrumentales, las actitudes punitivas encontrarían su origen en las percepciones y vivencias particulares, aflorando en aquellos casos en los que los sujetos identifican el delito como un riesgo posible para sí mismos y la comunidad de la que participan (Maruna y King, 2004; Tyler y Boeckman, 1997). Con objeto de verificar estos presupuestos, ha ido fraguándose un sólido cuerpo teórico, que bajo la denominación de "crime salience", ha explorado el rol que desempeña la problematización del delito en la opinión pública. Para ello, ha examinado el impacto de la experiencia de victimación (componente experiencial) y el miedo al delito (componente afectivo) en los juicios colectivos.

Con relación a la primera variable, a excepción de los limitados estudios que han encontrado una vinculación positiva entre victimación y punitivismo (Anderson, Bromley y Given, 2005; Haines, 2007; McAuley y McDonald, 2007), la literatura ha 
probado la independencia de ambos factores (Baron y Hartnagel, 1996; Covell y Howe, 1996; Gelb, 2006, 2008; Hartnagel y Templeton, 2012; Mahew y Van Kesteren, 2002; Payne y otros, 2004; Tufts y Roberts, 2002; Wood y Vicki, 2004).

De igual modo, los resultados referidos al efecto que genera el miedo al delito en las actitudes se han caracterizado por su ambivalencia y variabilidad (Mahew y Van Kesteren, 2002), revelando que éste constituye un elemento consustancial al delito que, en cierta medida, es independiente de las tasas de criminalidad y victimación existentes (Garland, 2001). A pesar de ello, los hallazgos obtenidos han evidenciado que quienes se perciben a sí mismos como potenciales víctimas son más propensos a expresar postulados retributivos (Barber y Doob, 2004; Costelloe, Chiricos y Gertz, 2009; Gelb, 2008; Hartnagel y Templeton, 2012; Mahew y Van Kesteren, 2002; Schwartz y otros, 1993). En este sentido, es destacable el efecto indirecto que desempeñan los medios de comunicación sobre las actitudes punitivas a través de un doble mecanismo que comprende tanto la preocupación por la delincuencia como el miedo al delito (Gelb, 2011; Goidel, Freeman y Procopio, 2006; Pfeiffer, Windzio y Kleimann, 2005).

Aunque las variables instrumentales han mostrado su potencial para mediar la opinión pública, su capacidad explicativa ha sido muy limitada, clarificando entre el 1,3\% y el 4\% de la varianza en las actitudes punitivas (Gelb, 2011; Maruna y King, 2004; King y Maruna, 2010; Spiranovic y otros, 2012).

\section{e. Variables expresivas}

En paralelo al desarrollo de las tesis instrumentales y como complemento de ellas fueron gestándose las teorías expresivas, unificadoras de una variedad de enfoques que han relacionado las actitudes punitivas con la inseguridad ontológica (Giddens, 1990, citado en Garland, 2001). Esto es, la inquietud generalizada acerca de las condiciones sociales, el debilitamiento de la cohesión social y la erosión de los valores tradicionales que ha caracterizado la posmodernidad. Estas aproximaciones han examinado el significado simbólico de la conducta delictiva, enlazando las reacciones sociales hacia el delito con la ansiedad por las circunstancias estructurales imperantes. Sugiriendo, así, que el punitivismo no obedece a motivos utilitarios -la disuasión de los infractores reales y potenciales-, sino que surge con el propósito de proteger y reafirmar las normas 
sociales, para las cuales el delito supone una afronta (Unnever y Cullen, 2010). En este ámbito, los predictores más relevantes del sentimiento punitivo han sido la intolerancia racial y étnica, la inseguridad económica, la ansiedad acerca de los valores y principios morales de la juventud, el rechazo hacia las políticas de bienestar social y la menor confianza interpersonal de los ciudadanos (Hogan, Chiricos y Gertz, 2005; King y Maruna, 2010; Kornhauser, 2013; Maruna y King, 2008; Unnever y Cullen, 2010). Los resultados alcanzados por este corpus teórico han constatado que las variables expresivas son poderosos predictores de la opinión pública -superiores al miedo al delito y la experiencia de victimación (Van Marle y Maruna, 2010; Tyler y Boeckmann, 1997)-, cuya capacidad explicativa ha fluctuado entre el 16\% y el 17\% de la varianza en las actitudes punitivas (King y Maruna, 2010; Maruna y King, 2004), asumiendo un tamaño de efecto mediano en las investigaciones de esta índole (Cohen, 1998).

\section{Síntesis}

A pesar de las inconsistencias entre unos y otros estudios, la literatura analizada ha permitido delimitar un conjunto de factores que, directa e indirectamente, condicionan las actitudes hacia el castigo juvenil. Siendo todos ellos significativos, su capacidad predictiva no ha sido homogénea, pudiendo distinguirse dos grandes grupos atendiendo a su potencial explicativo. El primero, aglutinador de los predictores débiles de las actitudes, acoge a las variables demográficas, atribucionales e instrumentales. Dentro de ellas, el nivel educativo, la orientación política, el estilo atribucional clásico y el miedo al delito han mostrado ser los factores explicativos más importantes. El segundo grupo, integrador de los predictores moderados de las actitudes, reúne a las variables cognitivas y aquellas que vinculan el punitivismo con elementos subjetivos de preocupación por los procesos de descomposición social y transformación de los valores convencionales. Bajo este marco, el conocimiento de la criminalidad juvenil, la justicia de menores y las particularidades del delito, así como la comprensión del desarrollo evolutivo de los adolescentes constituyen los factores más relevantes. Todo ello ilustra la variedad de elementos, mutuamente relacionados, que contribuyen a explicar el sentimiento punitivo y dan cuenta de las diferencias individuales. 


\section{Principales métodos y técnicas de investigación}

La creciente influencia adquirida por la opinión pública, con su consiguiente repercusión en la evolución de la política criminal, indujo el desarrollo exponencial de este ámbito de estudio. Inicialmente, los trabajos que exploraron las actitudes hacia la delincuencia juvenil y las respuestas ofrecidas a los menores fueron realizados mediante elementales instrumentos de medida que imposibilitaban la comprensión integral de las preferencias ciudadanas, así como la apreciación de sus diversos matices (Cullen, Fisher y Applegate, 2000). Los mismos comprendían cuestionarios compuestos por preguntas genéricas, de carácter abstracto, cuyas respuestas tendían a la representación de una ciudadanía punitiva (Spiranovic y otros, 2012; Tufts y Roberts, 2002; Tyler y Boekmann, 1997; Wood y Vicki, 2004). Y ello era así porque la imprecisión en la formulación de las preguntas, unida a la identificación social de la delincuencia juvenil con la delincuencia violenta, provocaban que la población imaginara los supuestos más graves -aunque menos representativos- al emitir sus respuestas, expresando, así, posicionamientos retributivos (Applegate y otros, 2009; Bishop, 2006; Gelb, 2011; Hough y Roberts, 2004; Roberts y Stalans, 1998; Stalans, 2002; Tufts y Roberts, 2002).

Tales hallazgos condujeron a la redefinición de los cuestionarios iniciales, incorporando a las preguntas tradicionales dos procedimientos complementarios orientados a superar estas deficiencias. El primero de ellos fue la denominada técnica del caso-escenario, consistente en la formulación de supuestos concretos, dotados de información detallada acerca del hecho delictivo y del infractor, que prevenían las respuestas basadas en estereotipos e imágenes distorsionadas (Applegate y otros, 2009; Bishop, 2006; Gelb, 2011; Hamilton y Harvey, 2005; Varona, 2008) ofreciendo una perspectiva más rigurosa de las actitudes. Más recientemente, se han desarrollado las encuestas de valoración económica (contingent valuation surveys), a través de las cuales se solicita a los encuestados que expresen la cuantía que estarían dispuestos a destinar a los distintos programas con el propósito de reducir la delincuencia (Jones y Weatherburn, 2011, Nagin, Piquero, Scott y Steinberg, 2006; Steinberg y Piquero, 2010).

A pesar del desarrollo y perfeccionamiento experimentados por esta técnica, que han convertido a la encuesta en el método de aproximación más utilizado (Gelb, 2006; 
Hamilton y Harvey, 2005; Roberts y Stalans, 1998), la misma no se halla exenta de limitaciones. Muestra de ello han sido las distintas voces que se han alzado advirtiendo de la ineptitud de este procedimiento para recoger la complejidad y el dinamismo de las actitudes, ofreciendo una óptica parcial de las mismas (Gelb, 2006; Green, 2006; Roberts, 2004; Stalans, 2002) e imposibilitando la distinción de las opiniones inconsistentes -que Converse (1964) conceptuaría como "non-attitudes" y Habermas (2006) como "mere opinion"- de los juicios reflexivos y estables (Gelb, 2006; Green, 2006, 2012; Marteache, 2012).

Todo ello motivó la coexistencia de estas aproximaciones con un cuerpo creciente de estudios conducidos mediante grupos de discusión (Gunnlaugsson, 2011; Hutton, 2005), cuyo desarrollo ha permitido una visión más precisa de las preferencias públicas, compensando los déficits de profundidad que comportaban las encuestas tradicionales. Sin embargo, este enfoque ha entrañado también impedimentos importantes que han sido documentados detalladamente en la literatura, siendo las carencias de validez externa su principal obstáculo (Gelb, 2006; Green, 2012; Krueger, 1988).

La encuesta deliberativa emergió entonces como instrumento híbrido entre ambas técnicas, combinando el potencial de generalización propio de la metodología cuantitativa, con la profundidad inherente a los análisis cualitativos (Fishkin, 1995; Marteache y otros, 2010). Asentada en la distinción entre opinión pública simple caracterizada por su volatilidad- y opinión pública meditada -caracterizada por su consistencia y estabilidad-, la encuesta deliberativa pretendía perfeccionar el abordaje metodológico de este objeto de estudio. Las restricciones que se le atribuyen comprenden el limitado repertorio de contenidos abordables en cada trabajo, su elevado coste de implementación, así como el hecho de que la naturaleza de los cambios actitudinales queda supeditada a la tipología de información que se proporciona a los participantes (Esmée Fairbain Foundation, 2002; Gelb, 2006; Haines y Case, 2007; Roberts y Stalans, 1998).

Esta breve revisión refleja la considerable transformación que han experimentado los métodos de medición de las actitudes, que va desde la aplicación de instrumentos rudimentarios, con restringida validez psicométrica, hasta el empleo de herramientas 
cuidadosamente validadas que proporcionan datos susceptibles de análisis multivariantes, con un amplio respaldo empírico.

\section{Implicaciones teóricas y metodológicas}

Una vez completado el análisis de la literatura y como conclusión de éste, se recogen las principales implicaciones teóricas y metodológicas que se desprenden de la revisión efectuada.

(i) Considerando que el estudio de la opinión pública hacia la delincuencia juvenil se ha desarrollado principalmente en Estados Unidos, Canadá y Reino Unido (Gelb, 2006; Roberts, 2004; Spiranovic y otros, 2012), la primera de estas aportaciones acentúa la necesidad de expandir la investigación a otras regiones. Y ello es así porque el conocimiento acumulado deriva de los hallazgos obtenidos en estos países. Dado que las particularidades de los sistemas de justicia juvenil dificultan la extrapolación de los resultados (Trzcinski y Allen, 2012), y en tanto que éste es un ámbito apenas cultivado en diversos países, la expansión de este cuerpo científico emerge como elemento destacado. Junto a ello, adquiere especial relevancia la articulación de estudios comparativos que permitan obtener una representación completa de las actitudes, que revele tanto sus rasgos comunes como las particularidades propias de cada realidad ${ }^{3}$.

(ii) En segundo lugar, cabe señalar las potencialidades que comporta la triangulación metodológica, atendiendo a dos circunstancias fundamentales. La primera de ellas alude a las limitaciones inherentes a cada técnica de investigación (Hutton, 2005; Roberts y Indermaur, 2007; Stalans, 2002), mientras que la segunda se refiere al carácter complejo de la opinión pública (Mays y Rudell, 2012; Mears y otros, 2007; Roberts, 2004; Steinberg y Piquero, 2010). Por ello, la elección de un enfoque multimétodo permitirá superar los déficits propios de cada procedimiento, ofreciendo una imagen más rigurosa y comprehensiva de las percepciones ciudadanas (Gelb, 2006, 2008; Perelman y Clements, 2009; Roberts y Stalans, 1998; Scott y otros, 2006).

\footnotetext{
${ }^{3}$ Ciertamente, los estudios comparativos han sido una práctica infrecuente en este ámbito (Jones y Weatherburn, 2011; Mahew y Van Kesteren, 2002), reflejando, en cierto modo, las dificultades que entraña aplicar procedimientos estandarizados en países distintos regidos por legislaciones diferentes.
} 
(iii) La tercera de las conclusiones formuladas profundiza en la exigencia de perfeccionar los procedimientos de medición de las actitudes, tratando de evitar el estudio de lo que se ha dado en llamar "non-attitudes" (Converse, 1964) o "mere opinion" (Habermas, 2006) -esto es, aquellas posiciones caracterizadas por su inconsistencia y superficialidad-, cuya medición ha contribuido a apoyar el populismo punitivo (Roberts, Stalans, Indermaur y Hough, 2003; Minkes, 2007). En la medida en que el cuestionario constituye la técnica de recogida de datos empleada en mayor medida (Gelb, 2006; Hamilton y Harvey, 2005; Roberts y Stalans, 1998), resulta esencial avanzar en su configuración y aplicación a la medición de las actitudes, combinando las preguntas generales con los casos-escenario y las valoraciones económicas, logrando así una visión más precisa y matizada de las preferencias públicas (Applegate y otros, 2009; Gelb, 2008; Roberts y otros, 2003). Para ello, han de abordarse dos cuestiones fundamentales que comprenden la especificación del constructo punitivismo y su evaluación a través de instrumentos cuyas propiedades métricas hayan sido cuidadosamente validadas. El primer requerimiento obedece a la diversidad de modos en que ha sido operativizada la variable punición ${ }^{4}$ (Roberts y Hasting, 2007), siendo necesario homogeneizarlos con la finalidad de poder contrastar los resultados y progresar en la sistematización de las evidencias. Adicionalmente, este proceso exige la elaboración de escalas de medida. El motivo es que aunque tradicionalmente el punitivismo fue cuantificado a través de ítems aislados, su naturaleza compleja aconseja la utilización de indicadores múltiples que permitan recoger las distintas dimensiones que encierra, reduciendo los errores de medida y obteniendo una perspectiva más completa del mismo (Gelb, 2008; Oppenheim, 2005). Finalmente, la validación de estos instrumentos, que avale empíricamente sus propiedades de validez y fiabilidad constituye una condición fundamental, que adquiere mayor relevancia en nuestro contexto, al carecer de herramientas específicas que hayan superado este procedimiento y puedan replicarse con garantías.

\footnotetext{
${ }^{4}$ Que incluye, entre sus principales exponentes, el requerimiento de condenas más severas (Grasmick y McGill, 1994; Doob, 2000; Scott y otros, 2006; Costelloe y otros, 2009), la delimitación del castigo como objetivo principal de la pena (Hough y Roberts, 2004; Haines, 2007; Haines y Case, 2007), la preferencia por las medidas privativas de libertad (Grasmick y McGill, 1994; Mayhew y Van Kesteren, 2002; Tufts, 2002) y el procesamiento de los menores en tribunales ordinarios, la imposición de condenas de adultos y su cumplimiento en dependencias comunes (Mears, 2001; Costelloe y otros, 2009; Steinberg y Piquero, 2010; Allen y otros, 2012; Trzcinski y Allen, 2012).
} 
(iv) La siguiente aportación se adentra en el abordaje de las actitudes punitivas como objeto de estudio. Y ello es así porque a la desigualdad en la operacionalización de las variables se une el hecho de que la mayoría de estudios han focalizado su atención en aspectos concretos de este cuerpo teórico -fundamentalmente el posicionamiento hacia la transferencia de los menores a la jurisdicción de adultos, la ejecución de jóvenes o la aceptación de la educación o el castigo como principios vehiculares de la justicia juvenil-, dificultando la articulación de una perspectiva integral de la opinión ciudadana (Mandracchia, Shaw y Morgan, 2013). Por ello, resulta esencial avanzar en la medición de los distintos componentes que conforman el punitivismo, previniendo sus mediciones parciales y facilitando la comparación de resultados. En este mismo sentido, el análisis de la opinión pública hacia los distintos principios que rigen la justicia de menores constituye uno de los ejes fundamentales del desarrollo de esta línea de investigación. Puesto que la evidencia acumulada ha rechazado la dicotomía rehabilitación-castigo (Mascini y Houtman, 2006; Moon y otros, 2000) y reconociendo que los trabajos que exploran exclusivamente las actitudes retributivas no permiten evaluar la medida en que la ciudadanía apoya otras medidas de control del delito (Turner, Cullen, Sundt y Applegate, 1997), su evaluación conjunta contribuiría a superar la parcialidad que caracterizó las aproximaciones previas, respondiendo a la complejidad propia de este ámbito de estudio.

(v) De otro lado, es destacable la necesidad de ahondar en las vinculaciones existentes entre las actitudes y sus factores explicativos, profundizando en la comprensión de las relaciones de causalidad y/o correlación entre unas y otros (Mears, 2001; Perelman y Clements, 2009). En el momento actual de desarrollo de este cuerpo teórico, la mayoría de estudios han indicado asociaciones entre variables (Mears, 2001). Esta circunstancia acentúa la necesidad de desarrollar diseños experimentales y realizar análisis multivariantes que permitan inferir relaciones causales. De igual modo, pese a la diversidad de trabajos que han explorado el efecto de determinadas variables, la interdependencia que existe entre ellas -que llega a anular el potencial explicativo de algunos factores al controlar el efecto de otros (Applegate y otros, 2009; Roberts y Indermaur, 2007)- acrecienta la necesidad de estudiarlas en interacción, elaborando 
modelos explicativos y valorando la contribución relativa de cada una de ellas a la explicación integral de las actitudes ${ }^{5}$ (Spiranovic y otros, 2012).

(vi) La última conclusión, que enfatiza la utilidad de desarrollar estudios longitudinales, obedece a la amplia publicidad mediática que provocan algunos delitos, que aun siendo muy graves, no son representativos de la criminalidad juvenil. Por ello, la valoración del impacto de la coyuntura concreta en los resultados obtenidos constituye un aspecto de especial trascendencia, pues las mediciones realizadas en momentos de agitación motivados por la sucesión de casos altamente mediatizados, son propensas a reflejar una excitación emocional intensa y no las preferencias estables de los participantes (Hohl y otros, 2013; Jan y otros, 2008; Scott y otros, 2006). De este modo, el desarrollo de investigaciones longitudinales, destinadas a examinar las actitudes de la población en el curso del tiempo, facilitará la superación de estos sesgos, ofreciendo mediciones más rigurosas de las mismas, que muestren tanto sus rasgos distintivos como sus variaciones circunstanciales.

Estas conclusiones, lejos de ser una relación estática o definitiva de recomendaciones, pretenden contribuir a la producción de este ámbito de estudio, aportando elementos útiles para su configuración y desarrollo futuros. En la medida en que el legislador se ha apoyado en las demandas sociales para justificar la reciente deriva punitiva, resulta esencial conocer con exactitud qué reclama la ciudadanía. La noción de una opinión pública compacta, monolítica y esencialmente punitiva, que arrojaron estudios con importantes limitaciones metodológicas (Cullen y otros, 2000; Roberts y Stalans, 1997), se encuentra hoy superada. Sin embargo, el conocimiento acumulado es parcial y la vinculación entre los sentimientos colectivos y las reformas adoptadas continúa siendo incierta. Por ello, el incremento y perfeccionamiento de las investigaciones en esta materia resulta imprescindible, tanto para prevenir la perpetuación de concepciones erróneas, como para identificar las discrepancias existentes entre las reclamaciones ciudadanas y las políticas impulsadas.

\footnotetext{
${ }^{5}$ Aspecto, éste último, particularmente importante, teniendo en cuenta las limitaciones que entrañan las comparaciones inter-estudios.
} 


\section{Agradecimientos}

Este trabajo se ha realizado con el apoyo económico del Programa de Formación del Profesorado Universitario (FPU) a cargo del Ministerio de Educación. Agradezco a mi directora de tesis, compañera y amiga Esther Fernández Molina su asesoramiento, sus sugerencias y su inestimable apoyo durante este proceso y a los investigadores Casimiro Barbado y David Vázquez sus comentarios al manuscrito. Finalmente, agradezco al editor y a los revisores anónimos de la REIC las aportaciones realizadas que han permitido mejorar la calidad científica del trabajo.

\section{Referencias}

Aizpurúa, E. y Fernández, E. (2011). Información, ¿Antídoto frente al "populismo punitivo"?. Revista Española de Investigación Criminológica, 3(9). Disponible en http://www.criminologia.net/pdf/reic/ano9-2011/a92011art3.pdf

Allan, J., Edwards, S., Patterson, A. y Smith, D. (2006). Policing and the criminal justice system. Home Office Online Report 07/06. Disponible en http://tna.europarchive.org/20100419081706/http://www.homeoffice.gov.uk/rds/pdf s06/rdsolr0706.pdf

Allen, R. (2002). "There must be some way of dealing with kids": Young offenders, public attitudes and policy change. Youth Justice, 2(1), 3-13.

Allen, T.T., Trzcinscki, E. y Kubiak, S.P. (2012). Public attitudes toward juveniles who commit crimes: The relationship between assessments of adolescent development and attitudes toward severity of punishment. Crime \& Delinquency, 58(1), 78-102.

Anderson, S., Bromley, C. y Given, L. (2005). Public attitudes toward young people and youth crime in Scotland: findings from the 2004 Scottish social attitudes survey. Edinburgh: Scottish Executive Education Department.

Applegate, B.K. y Davis, R.K. (2006). Public views on sentencing juvenile murderers. The impact of offender, offense and perceived maturity. Youth Violence \& Juvenile Justice, 4(1), 55-74.

Applegate, B.K., Davis, R.K. y Cullen, F.T. (2009). Reconsidering child saving: The extent and correlates of public support for excluding youths from the juvenile court. Crime Delinquency, 55(1), 51-77.

Barber, J. y Doob, A.N. (2004). An analysis of public support for severity and proportionality in the sentencing of youthful offenders. Canadian Journal of Criminology and Criminal Justice, 46(3), 327-342.

Baron, S. y Hartnagel, T.F. (1996). "Lock 'em up": Attitudes toward punishing juvenile offenders. Canadian Journal of Criminology, 38(2), 191-212. 
Bishop, D.M. (2006). Public opinion and juvenile justice policy: Myths and misconceptions. Criminology \& Public Policy, 5(4), 653-664.

Bottoms, A. (1995). The philosophy and politics of punishment and sentencing. En C. Clarkson y R. Morgan (Eds.), The Politics of Sentencing Reform (Pp. 17-50). Oxford: Clarendon Press.

Carmichael, J.T. y Burgos, G. (2012). Sentencing juvenile offenders to life in prison: The political sociology of juvenile punishment. American Journal of Criminal Justice, 37(4), 602-629.

Chapman, B., Mirrlees-Black, C. y Brawn, C. (2002). Improving public attitudes to the Criminal Justice System: The impact of information. London: Home Office.

Cochran, J.K., Boots, D.P. y Heide, K.M. (2003). Attribution styles and attitudes toward capital punishment for juveniles, the mentally incompetent, and mentally retarded. Justice Quarterly, 20(1), 65-93.

Cohen, J. (1998). Statistical power analysis for the behavioral sciences. New York: Academic.

Converse, P.E. (1964). The nature of belief systems in mass publics. En D.E. Apter (Ed.), Ideology and discontent. (Pp. 206-261). London: Free Press of Glencoe.

Costelloe, M.T., Chiricos, T. y Gertz, M. (2009). Punitive attitudes towards criminals. Exploring the relevance of crime salience and economic insecurity. Punishment \& Society, 11(1), 25-49.

Covell, K. y Howe, B. (1996). Public attitudes and juvenile justice in Canada. The International Journal of Children's Rights, 4(3), 345-355.

Cullen, F.T., Fisher, B.S. y Applegate, B.K. (2000). Public opinion about punishment and corrections. En M. Tonry (Ed.), Crime and Justice: A Review of Research, 27. (Pp. 1-79). Chicago: University of Chicago Press.

Esmée Fairbain Foundation (2002). Attitudes to crime and punishment: The results of a deliberative poll of public opinion. London: Autor.

Estrada, F. (2001). Juvenile violence as a social problem. Trends, media attention and societal response. British Journal of Criminology, 41(4), 639-655.

Evans, T.D. y Adams, M. (2003). Salvation or damnation: Religion and correctional ideology. American Journal of Criminal Justice, 28(1), 15-35.

Faucher, C. (2009). Fear and loading in the news: a qualitative analysis of Canadian print news coverage of youthful offending in the twentieth century. Journal of Youth Studies, 12(4), 439-456. 
Fernández, E. (2013). Valoración ciudadana del castigo juvenil. Barómetro Observatorio de la Actividad Judicial 2012. La Ley, 7944.

Fernández, E. y Tarancón, P. (2010). Populismo punitivo y delincuencia juvenil: Mito o realidad. Revista Electrónica de Ciencia Penal y Criminología, 12(8). Disponible en http://criminet.ugr.es/recpc/12/recpc12-08.pdf

Fishkin, J. (1995). Democracia y deliberación. Barcelona: Ariel.

Garberg, N.M. y Libkuman, T.M. (2009). Community sentiment and the juvenile offender: Should juveniles charged with felony murder be waived into de adult criminal justice system? Behavioral Sciences \& the Law, 27(4), 553-575.

García, E. (2013). ¿Los conocimientos criminológicos modulan la actitud punitiva? Estudios sobre el Mensaje Periodístico, 19(2), 753-761.

García, M.D., Martín, E., Torbay, A. y Rodríguez, C. (2010). La valoración social de la Ley de Responsabilidad Penal de los Menores. Psicothema, 22(4), 865-871.

Garland, D. (2001). The culture of control. Crime and social order in contemporary society. New York: Oxford University Press.

Gelb, K. (2006). Myths and misconceptions: Public opinion versus public judgment about sentencing. Melbourne: Sentencing Advisory Council.

Gelb. K. (2008). More myths and misconceptions. Melbourne: Sentencing Advisory Council.

Gelb, K. (2011). Predictors of punitiveness: Community views in Victoria. Melbourne: Sentencing Advisory Council.

Goidel, R.K., Freeman, C.M. y Procopio, S.T. (2006). The impact of television viewing on perceptions of juvenile crime. Journal of Broadcasting \& Electronic Media, 50(1), 119-139.

Grasmick, H.G. y McGill, A.L. (1994). Religion, attribution style and punitiveness toward juvenile offenders. Criminology, 32(1), 23-46.

Green, D.A. (2006). Public opinion versus public judgment about crime: Correcting the "comedy of errors". British Journal of Criminology, 46(1), 131-154.

Green, D.A. (2012). When children kill children. Penal populism and political culture. New York: Oxford University Press.

Guanarteme, F. (2009). Alarma social y derecho penal. InDret. Revista para el análisis del Derecho, 1. Disponible en http://www.indret.com/pdf/598.pdf 
Guetti, S. y Redlich, A.D. (2001). Reactions to youth crime: perceptions of accountability and competency. Behavioral Sciences \& the Law, 19(1), 33-52.

Gunnlaugsson, H. (2011). Public attitudes to punishment. Iceland: Scandinavian Research Council for Criminology.

Habermas, J. (2006). The public sphere: An encyclopedia article. En M.G. Durham y D.M. Kellner (Eds.), Media and cultural studies. Keyworks. (Pp. 73-78). Cornwall: Blackwell Publishing.

Haines, A. (2007). Juvenile crime and punishment in Bucharest, Romania: A public opinion survey. Internet Journal of Criminology. Disponible en http://www.internetjournalofcriminology.com/Haines $\% 20$ \%20Juvenile\%20Crime\%20and\%20Punishment $\% 20$ in\%20Bucharest.pdf

Haines, K. y Case, S. (2007). Individual differences in public opinion about youth crime and justice in Swansea. The Howard Journal, 46(4), 338-355.

Halsey, K. y White, R. (2008). Young people, crime and public perceptions: a review of the literature. Slough: National Foundations for Educational Research.

Hamilton, C. y Harvey, R. (2005). The role of statistics and public opinion in the implementation of international juvenile justice standards. Helsinki: European Institute for Crime Prevention and Control.

Hartnagel, T.F. y Templeton, L.J. (2012). Emotions about crime and attitudes to punishment. Punishment \& Society, 14(4), 452-474.

Hogan, M.J., Chiricos, T. y Gerzt, M. (2005). Economic insecurity, blame, and punitive attitudes. Justice Quarterly, 22(3), 392 - 412.

Hohl, K., Satnko, B. y Newburn, T. (2013). The effect of the 2011 London disorder on public opinion of police and attitudes towards crime, disorder, and sentencing. Policing, 7(1), 12-20.

Hough, M. y Park, A. (2002). Attitudes to crime and punishment: The results of a deliberative poll of public opinion. London: Esmée Fairbairn Foundation.

Hough, M. y Roberts, J.V. (1998). Attitudes to punishment: Findings from the British Crime Survey. London: Home Office.

Hough, M. y Roberts, J.V. (2004). Youth crime and youth justice. Public opinion in England and Wales. Bristol: The Policy Press.

Hutton, N. (2005). Beyond populist punitiveness? Punishment \& Society, 7(3), 243258. 
Indermaur, D., Roberts, L., Spiranovic, C., MacKenzie, G. y Gelb, K. (2012). A matter of judgement: The effect of information and deliberation on public attitudes to punishment. Punishment \& Society, 14(2), 147-165.

Jan, I.F., Ball, J.D. y Walsh, A. (2008). Predicting public opinion about juvenile waivers. Criminal Justice Policy Review, 19(3), 285-300.

Jones, C.A. y Weatherburn, D.J. (2011). Willingness to pay for rehabilitation versus punishment to reduce adult and juvenile crime. Australian Journal of Social Issues, 46(1), 9-27.

King, A. y Maruna, S. (2010). Is a conservative just a liberal who has been mugged? Exploring the origins of punitive views. Punishment \& Society, 11(2), 147-169.

Kornhauser, R. (2013). Reconsidering predictors of punitiveness in Australia: A test of four theories. Australian \& New Zealand Journal of Criminology, 46(2), 221-240.

Krueger, R.A. (1988). Focus groups: A practical guide for applied research. California: Sage.

Mahew, P. y Van Kesteren, J. (2002). Cross-national attitudes to punishment. En J.V. Roberts y M. Hough (Eds.), Changing attitudes to punishment. Public opinion, crime and justice. (Pp. 63-92). Devon: Willan Publishing.

Mandracchia, J.T., Shaw, L.B. y Morgan, R.D. (2013). What's with the attitude: Changing attitudes about criminal justice issues. Criminal Justice \& Behavior, 40(1), 95-113.

Marteache, N. (2012). Deliberative processes and attitudes toward sex offenders in Spain. European Journal of Criminology, 9(2), 159-175.

Marteache, N., Martínez, M. y Pérez, M. (2010). Comparación entre opinión pública y "opinión pública meditada" en relación a la delincuencia sexual. Revista Española de Investigación Criminológica, 7(8). Disponible en http://www.criminologia.net/pdf/reic/ano8-2010/a82010art7.pdf

Maruna, S. y King, A. (2004). Public opinion and community penalties. En T. Bottoms, S. Rex y G. Robinson (Eds.), Alternatives to prison: Options for an insecure society. (Pp. 83-112). Cullompton: Willan.

Maruna, S. y King, A. (2008). Giving up on the young: A commentary. Current Issues in Criminal Justice, 20(1), 129-134.

Mascini, P. y Houtman, D. (2006). Rehabilitation and repression. Reassessing their ideological embeddedness. British Journal of Criminology, 46(5), 822-836.

Mays, G.L. y Ruddell, R. (2012). Do the crime, do the time. Juvenile criminals and adult justice in the American Court System. California: ABC-CLIO. 
McAuley, M. y McDonald, K.I. (2007). Russia and youth crime. A comparative study of attitudes and their implications. British Journal of Criminology, 47(1), 2-22.

Mears, D.P. (2001). Getting tough with juvenile offenders. Explaining support for sanctioning youths as adults. Criminal Justice \& Behavior, 28(2), 206-226.

Mears, D.P., Hay, C., Gertz, M. y Mancini, C. (2007). Public opinion and the foundation of the juvenile court. Criminology, 45(1), 223-257.

Moon, M.M., Sundt, J.L., Cullen, F.T. y Wright, J.P. (2000). Is child saving dead? Public support for juvenile rehabilitation. Crime \& Delinquency, 46(1), 38-60.

Nagin, D.S., Piquero, A.R., Scott, E.S. y Steinberg, L. (2006). Public preferences for rehabilitation versus incarceration of juvenile offenders: Evidence from a contingent valuation survey. Crime \& Public Policy, 5(4), 301-326.

Oppenheim, A.N. (2005). Questionnaire design, interviewing and attitude measurement. New York: Continuum.

Payne, B.K., Gainey, R.R., Triplett, R.A. y Danner, M.J.E. (2004). What drives punitive beliefs?: Demographic characteristics and justifications for sentencing. Journal of Criminal Justice, 32(3), 195-206.

Perelman, A.M. y Clements, C.B. (2009). Beliefs about what works in juvenile rehabilitation. The influence of attitudes on support for "get tough" and evidencebased interventions. Criminal Justice \& Behavior, 36(2), 184-197.

Pfeiffer, C., Windzio, M. y Kleimann, M. (2005). Media use and its impacts on crime perception, sentencing attitudes and crime policy. European Journal of Criminology, 2(3), 259-285.

Piquero, A.R., Cullen, F.T., Unnever, J.D., Piquero, N.L. y Gordon, J.A. (2010). Never too late: Public optimism about juvenile rehabilitation. Punishment \& Society, 12(2), 187-207.

Quinton, P. (2011). The impact of information about crime and policing on public perceptions. The results of a randomized controlled trial. London: National Policing Improvement Agency.

Rechea, C., Fernández, E. y Benítez, M.J. (2004). Tendencias sociales y delincuencia. Informe de Investigación, $11 . \quad$ Disponible en http://www.uclm.es/centro/criminologia/pdf/informes/11_2004.pdf

Roberts, J.V. (2004). Public opinion and youth justice. En M. Tonry y A.N. Doob (Eds.), Crime and Justice: A review of research, 31. (Pp. 495-542). Chicago: University of Chicago Press. 
Roberts. J.V. y Hastings, R. (2007). Public opinion and crime prevention: A review of international findings. Institute for the Prevention of Crime Review, 1, $193-218$.

Roberts, L. y Indermaur, D. (2007). Predicting punitive attitudes in Australia. Psychiatry, Psychology \& Law, 14(1), 56-65.

Roberts, J.V. y Stalans, L.J. (1998). Crime, criminal justice and public opinion. En M. Tonry (Ed.), The Handbook of Crime and Punishment. (Pp. 31-57). New York: Oxford University Press.

Roberts, J.V., Stalans, L.J., Indermaur, D. y Hough, M. (2003). Penal populism and public opinion. Lessons from five countries. New York: Oxford University Press.

Schwartz, I.M., Guo, S. y Kerbs, J.J. (1993). The impact of demographic variables on public opinion regarding juvenile justice: Implications for public policy. Crime \& Delinquency, 39(1), 5-28.

Scott, E.S., Repucci, N.D., Antonishak, J. y DeGennaro, J.T. (2006). Public attitudes about the culpability and punishment of young offenders. Behavioral Sciences \& the Law, 24(6), 815-832.

Serrano, A. (2011). Actitudes sobre derechos fundamentales procesal-penales: Una dimensión inexplorada de la punitividad. Revista Electrónica de Ciencia Penal y Criminología, 13(5). Disponible en http://criminet.ugr.es/recpc/13/recpc13-05.pdf

Sharp, S.F., McGhee, M., y Hope, T. (2007). Predictors of support of legislation banning juvenile executions in Oklahoma. Justice Quarterly, 24(1), 133-155.

Singer, L. y Cooper, S. (2008). Inform, persuade and remind. An evaluation of a project to improve public confidence in the criminal justice system. London: Ministry of Justice.

Spiranovic, C.A., Roberts, L.D., Indermaur, D., Warner, K., Gelb, K. y MacKenzie, G. (2012). Pubic preferences for sentencing purposes: What difference does offender age, criminal history and offence type make? Criminology \& Criminal Justice, 12(3), 289-306.

Sprott, J.B. (1996). Understanding public views of youth crime and the youth justice system. Canadian Journal of Criminology, 38(3), 271-290.

Sprott, J.B. (1999). Are members of the public tough on crime? The dimensions of public "punitiveness". Journal of Criminal Justice, 27(2), 467-474.

Stalans, L.J. (2002). Measuring attitudes to sentencing. En J. Roberts y M. Hough (Eds.), Changing attitudes to punishment: public opinion, crime and justice. (Pp. 15-32). Cullompton: Willan. 
Steinberg, L. y Piquero, A.R. (2010). Manipulating public opinion about trying juveniles as adults: an experimental study. Crime \& Delinquency 56(4), 487-506.

Trzcinski, E. y Allen, T. (2012). Justice towards youth: Investigating the mismatch between current policy and public opinion. Children \& Youth Services Review, 34, 27-34.

Tufts, J. (2002). Public attitudes toward the Criminal Justice System. Juristat, 20(12).

Tufts, J. y Roberts, J.R. (2002). Sentencing juvenile offenders: Comparing public preferences and judicial practice. Criminal Justice Policy Review, 13(1), 46-64.

Turner, M.G., Cullen, F.T., Sundt, J.L. y Applegate, B.K. (1997). Public tolerance for community-based sanctions. The Prison Journal, 77(1), 6-26.

Tyler, T.R. (2003). Procedural justice, legitimacy, and the effective rule of law. En M. Tonry (Ed.), Crime and Justice: A review of Research, 30. (Pp.283-357). Chicago: University of Chicago Press.

Tyler, T.R. y Boeckmann, R.J. (1997). Three strikes and youre out, but why? The psychology of public support for punishing rule breakers. Law \& Society Review, $31(2), 237-266$.

Unnever, J.D. y Cullen, F.T. (2010). The social sources of americans' punitiveness: A test of three competing models. Criminology, 48(1), 99-129.

Van Marle, F. y Maruna, S. (2010). "Ontological insecurity" and "terror management". Linking two free-floating anxieties. Punishment \& Society, 12(1), 7-26.

Varma, K. N. (2006). Face-ing the ofender: Examining public attitudes toward young offenders. Contemporary Justice Review, 9(2), 175-187.

Varona, D. (2008). Ciudadanos y actitudes punitivas: Un estudio piloto de población universitaria española. Revista Española de Investigación Criminológica, 1(6). Disponible en http://www.criminologia.net/pdf/reic/ano6-2008/a62008art1.pdf

Varona, D. (2013). Percepción y elección del castigo en España: Resultados a partir de la Encuesta Social Europea. Cuadernos de Politica Criminal, 111(3), 145-193.

Vaughman, H. (2010). Psicología social. Madrid: Panamericana.

Wood, J. y Vicki, G.T. (2004). Public perceptions of crime and punishment. En J. Adler (Ed.), Forensic psychology concepts, debates and practice. (Pp.16-36). Oxon: Willan.

$\mathrm{Wu}$, B. (2000). Determinants of public opinion toward juvenile waiver decision. Juvenile \& Family Court Decision, 51(1), 9-22. 
Eva Aizpurúa es becaria FPU en el Centro de Investigación de Criminología de la Universidad de Castilla-La Mancha. Sus principales líneas de investigación comprenden la justicia juvenil y las actitudes hacia el castigo de los menores infractores. 